no less than ten different hospitals over a period of twenty-six months; had had various treatments, including faradism and massage, yet who, under an appropriate moral treatment, regained his voice completely in four days.

These cases are aphonic through purely psychic conditions, and, as I have shown, can, by an intense, persistent, and energetic psychotherapy, be cured and returned at once to their unit, not only a satisfaction to the patient himself, but at the same time a very material gain to the country.

\title{
A NOTE ON THE AFTER-TREATMENT OF AURAL AND NASAL OPERATIONS.
}

\author{
By G. Wilkinson, M.B., F.R.C.S., \\ Sheffield.
}

$l_{\mathrm{N}}$ his presidential address to the Section of Otology, ${ }^{1} \mathrm{Mr}$. Banks-Davis raised the question as to the best after-treatment for cases of "radical" mastoid operation. Like him, I have tried all the recognised methods. For the last twelve months I have been using a modification of the Carrel-Dakin "progressive sterilisation" method, which bas given me better results than any of the methods previously tried.

I complete the operation by making a downward turning plastic meatal flap, which has the advantage of leaving the antral cavity freely exposed. I insert a large, but not tightly fitting, open rubber tube through the enlarged meatus. It is important that the tube should not press tightly on the edges of the meatal incision, otherwise perichondritis of the pinna is apt to be set up. The retro-auricular wound is completely sutured. A single fold of aseptic gauze is carried round the pinna, but no dressing at all covers the meatal tube. The whole ear is covered by a perforated zinc shield, fixed in position by tapes tied round the head. The patient lies on the opposite side, and the nurse is instructed to run the Carrel-Dakin solution into the tube with a dropper every two hours. The shield is simply raised each time the wound is irrigated, and subsequently replaced. I have also used acri-flavine solution for irrigation. This also gives good results, but, I think, not quite so good as the Carrel-Dakin solution.

The figure shows the shape of the shield and the method by which it is fixed in position. It will be noticed that the edges of the perforated zine are covered by a split rubber drainage-tube. The shield is sterilised by boiling.

The irrigation is kept up for a fortnight, at the end of which time the wound is sterile. The drain-tube is then removed, and the meatal flap is found to be fixed in position, and the meatus well open. The post-auricular wound is usually healed. The further treatment consists in light packing of the meatus with a strip of bismuth gauze. At this stage the packing can be done quite painlessly. The shield is usually worn for six weeks to prevent infection of the wound from outside. The ear is usually about dry at the end of six weeks.

I use a similar shield after nasal operations (as shown in the figure). It has the great advantage of preventing infection of the nose from the outside by the patient's fingers or handkerehief. Before I began to use

'Journ. of Lartngol., Rhinol., and Otol., vol. xxxiii, p. 321. 
this shieid I never succeeded entirely in eliminating this source of infection, in spite of the provision of sterile swabs for the patient's use.

It appears to me there are two principal sources of infection to be guarded against in nasal operations: (1) infection from the outside, usually conveyed by the patient's fingers; (2) entry of vomited matter in to the nose during or subsequent to the operation. The use of the cage eiiminates the first danger. The second factor has to be reckoned with only in cases operated upon under general anæsthesia. I consider it a very real danger, and I have seen several cases of infection of the maxillary antrum following soiling of the nose by vomit. The tendency of the patient to vomit on partial recovery from anæsthesia seems to be increased by preliminary preparation of the nose by cocaine and adrenalin, and by operating in the sitting position.

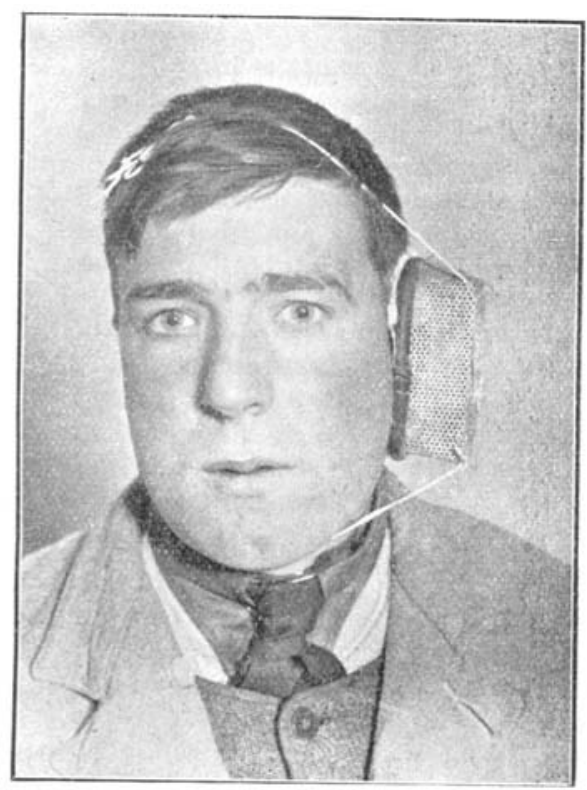

Cage for Carrel-Dakin Treatment of Mastoid Wound.

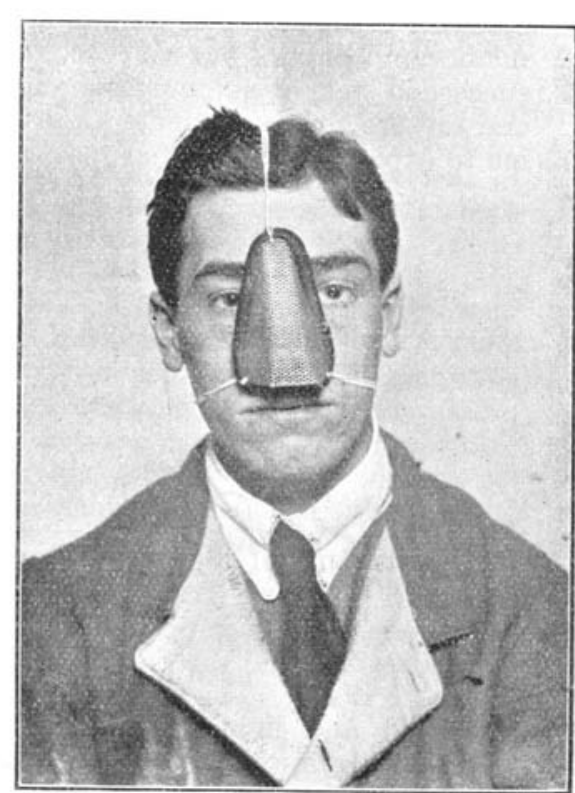

Cage for Use after Nasal Operations.

To prevent this accident, for several years I have made it a rule to employ intra-tracheal intubation, with sponge packing of the pharynx, in all nasal operations done under general anæsthesia-even submucous resections. The control of the anæsthesia is much better, and all danger of entry of blood, secretions, pus, or foreign bodies into the lungs is obviated, and also the danger of the nose becoming soiled by vomit is much reduced. At the conclusion of the operation I pack each nasal fossa with a rubber sponge tampon. The patient is rolled right. forward onto his face before the pharyngeal sponges and tracheal tube are removed. The nasal tampons are removed on the morning following the operation. The shield is worn for four to seven days.

Though outside the immediate scope of the title of this communication, I should like to refer to the good results obtained by the use of 
acri-flavin in cases of suppuration of the maxillary antrum. I have used it in six cases in the following manner: After puncturing the antrum I wash out with a mild alkaline solution or a solution of boric acid, and afterwards dry the cavity by blowing air through. I then lateralise the patient's head, and run in through the cannula one or two drachms of warm 1 in 1000 solution of acri-flavine. The head is kept on the side, slightly tilted backwards, for five minutes, and then bent forwards to allow the excess of solution to run out of the nose. At the next washing, two or three days later, one obtains one large "blob" of transparent yellow-stained mucus, mixed with flocculi of opaque orange-stained pus. The lavage is repeated at intervals until the yellow blob is obtained free from the orange-stained pus element. The acri-flavine is then discontinued, and at the next washing the antrum is usually found to be clear. I have not employed acri-flavine up to the present in any oldstanding cases with profuse suppuration and thickened polypoid mucous membrane lining, but one of the cases in which the method has succeeded was of six months, and another of three months' duration, and my previous experience of treatment by lavage would not have led me to expect a cure by that method alone without the acri-flavine.

\title{
SOCIETIES' PROCEEDINGS.
}

\author{
ROYAL SOCIETY OF MEDICINE-LARYNGOLOGICAL \\ SECTION. \\ June 1, 1917. \\ President: $\overline{\text { Mr. T. MARK Hovell. }}$
}

Treatment of Primary Carcinoma of the Nasopharynx by Colloidal Copper. - Irwin Moore.-Members will rem. mber that this case, a female, aged seventy, was shown at the meeting of the Section on November 3, 1916, three and a half months after removal of the growth (July 14, 1916). The left tonsil had enlarged since the operation, was congested and indurated, whilst patient complained of severe pains extending from the left side of the neck to the top of the head. No enlarged glands could be felt. Opinions were invited as to the question of recurrence in the tonsil, and advisability of enucleation.

In the meantime patient consulted her physician, who recommended injections of colloidal copper.

Patient is now exhibited, six and a half months later, to show the remarkable results of this treatment, which has apparently not only cured the pain, but also reduced the size and induration of the tonsil.

It is now ten months since removal of the growth, and there is no recurrence nor enlargement of glands in the neck.

Lupoid Tuberculosis of the Pharynx, affecting the Soft Palate and Uyula, in a Boy, aged eight, the subject of Congenital Syphilis.-Irwin Moore.-This case was shown at the Section meeting on March 2, 1917, as lymphadenoma, but in consequence of the discussion and the chronic character of the infiltration, together with the more recent clinical and pathological examinations, the title has been changed.

1 Jodrn. of Laryngol., Rhinol., and Otol., vol. xxxii, p. 103. 